\title{
Two-Step Multi-Physics Analysis of an Annular Linear Induction Pump for Fission Power Systems
}

\author{
Steven M. Geng ${ }^{1}$ and Dr. Terry V. Reid ${ }^{1}$ \\ NASA Glenn Research Center, Cleveland, Ohio, 44135
}

\begin{abstract}
One of the key technologies associated with fission power systems (FPS) is the annular linear induction pump (ALIP). ALIPs are used to circulate liquid-metal fluid for transporting thermal energy from the nuclear reactor to the power conversion device. ALIPs designed and built to date for FPS project applications have not performed up to expectations. A unique, two-step approach was taken toward the multi-physics examination of an ALIP using ANSYS Maxwell 3D and Fluent. This multi-physics approach was developed so that engineers could investigate design variations that might improve pump performance. Of interest was to determine if simple geometric modifications could be made to the ALIP components with the goal of increasing the Lorentz forces acting on the liquid-metal fluid, which in turn would increase pumping capacity. The multi-physics model first calculates the Lorentz forces acting on the liquid metal fluid in the ALIP annulus. These forces are then used in a computational fluid dynamics simulation as (a) internal boundary conditions and (b) source functions in the momentum equations within the Navier-Stokes equations. The end result of the two-step analysis is a predicted pump pressure rise that can be compared with experimental data.
\end{abstract}

\section{Nomenclature}

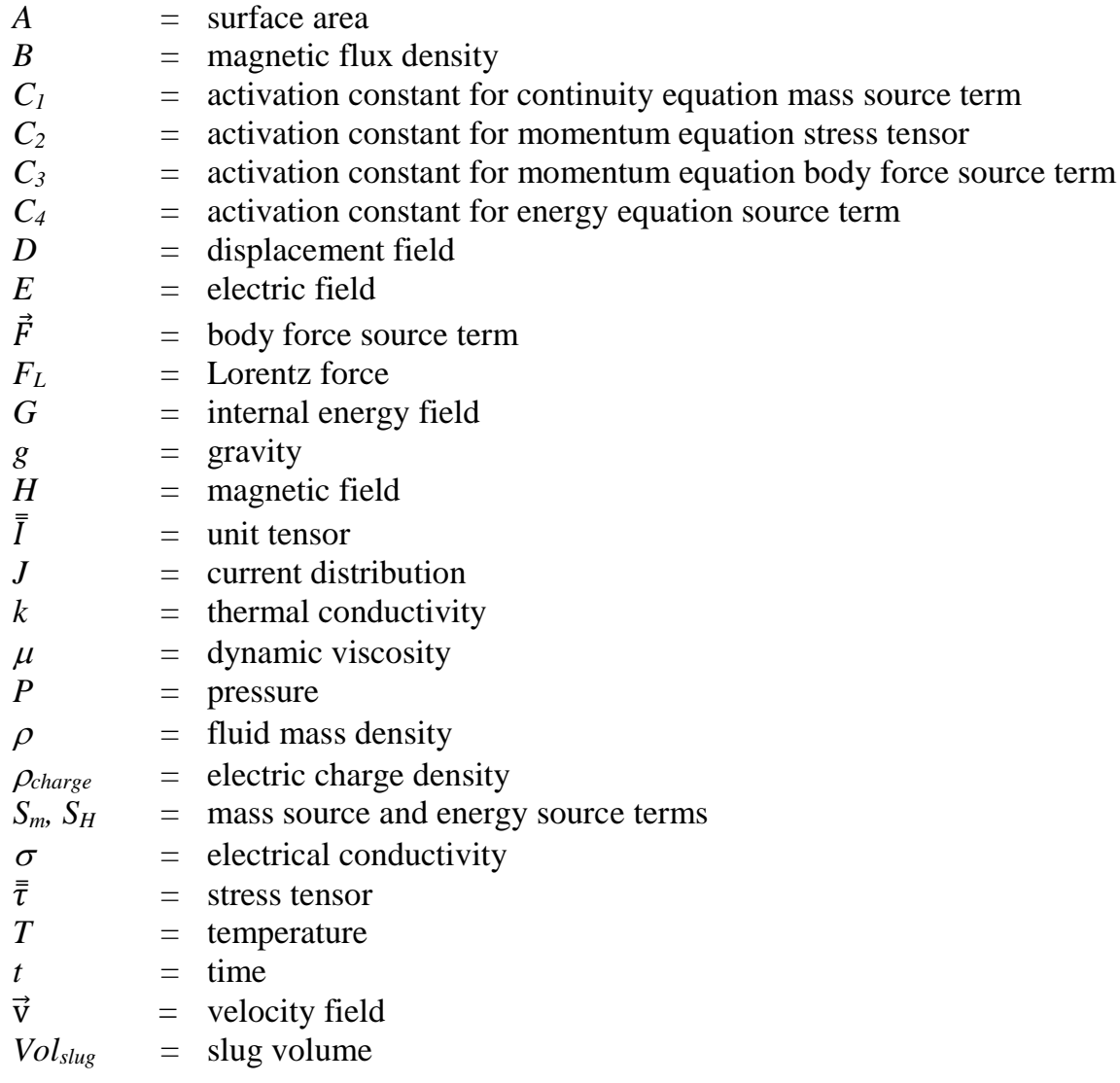

${ }^{1}$ Engineer, Thermal Energy Conversion Branch, 21000 Brookpark Road, M/S 301-2, AIAA Senior Member. 1

American Institute of Aeronautics and Astronautics 


\section{Introduction}

$\mathrm{F}$ ission power system (FPS) technology is being developed for use on the surface of the Moon, Mars, or other moons and planets of our solar system. FPSs are capable of providing good performance at any location, including those near the poles or other permanently shaded regions, and offer the capability to provide on-demand power at any time, even at long distances from the Sun. Fission-based systems also offer the potential for outposts, crew, and science instruments to operate in a power-rich environment.

One of the key technologies associated with the FPS is the annular linear induction pump (ALIP) used to circulate the liquid-metal fluid that transports thermal energy from the nuclear reactor to the power conversion device. In 2010, an ALIP developed by the Idaho National Laboratory (INL) was tested under representative space-reactor thermal operating conditions at NASA's Marshall Space Flight Center (MSFC) to quantify the pump's performance ${ }^{1}$. The measured performance was below expectations. Illustrations of the ALIP pump are shown in Figure 1. MSFC conducted performance testing of the ALIP using their test circuit shown in Figure 2. The test circuit consists of the ALIP, an induction heater, a throttling valve, an electromagnetic flow meter, and a gaseous nitrogen to NaK heat exchanger. The throttling valve is located downstream of the ALIP (in the test circuit) so that the flow resistance could be varied.
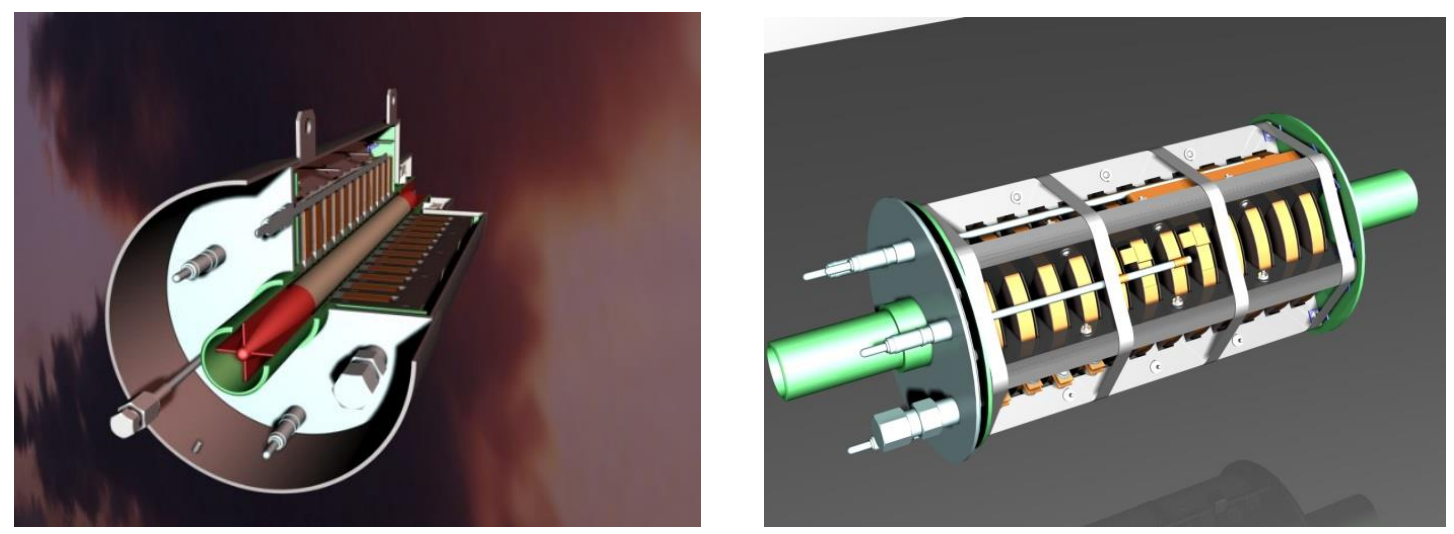

Figure 1. Illustrations of ALIP (Courtesy of Idaho National Lab)

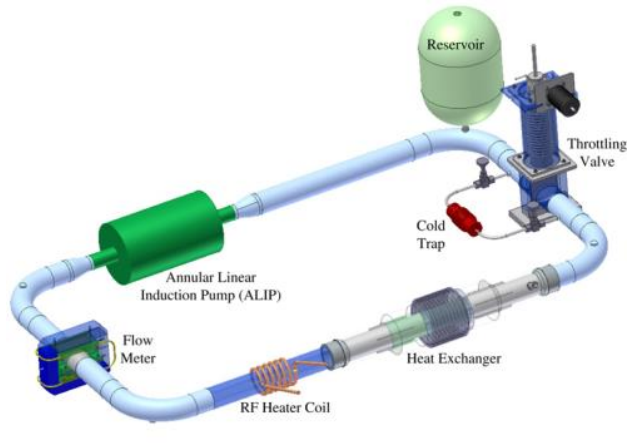

a) Schematic

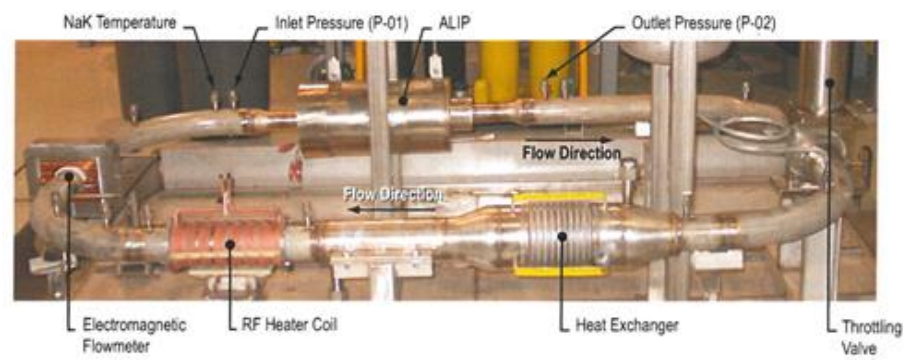

b) Photograph

Figure 2. The ALIP Test Circuit (Courtesy of NASA Marshall Space Flight Center)

In 2012, NASA's Glenn Research Center (GRC) developed a magneto-static finite element model of the ALIP to gain a better understanding of its functionality, and to independently evaluate design variations that might have contributed to the performance shortfall ${ }^{2}$. In 2015, the ALIP model was upgraded to a 3D transient magnetic analysis which dramatically simplified the post-processing of the model predictions. The magnetic model takes advantage of ALIP symmetry to reduce computation time.

A full 3D Computational Fluid Dynamics (CFD) model was recently created of the ALIP that, when used in combination with the magnetic model, could generate pressure rise and flow rate predictions for comparison with the 
experimental data. Symmetry is not assumed in the CFD model due to the inhomogenous pressure distribution in the NaK flow annulus.

The multi-physics ALIP model was validated against experimental data, then used to generate performance predictions. This paper presents a discussion of the multi-physics (3D transient magnetic and CFD) model of the ALIP, a comparison of model predictions versus experimental data, and a discussion of several design variations that could potentially have a positive impact on pump performance.

\section{Fission Power System Reference Concept Demonstration}

Power is an important consideration when planning the exploration of planetary surfaces. Nuclear power is an enabling option for locations in the solar system where sunlight is limited. A Fission Surface Power preliminary reference concept ${ }^{3}$ was developed, and a full scale, $1 / 4$ power Technology Demonstration Unit (TDU) was built and tested ${ }^{4}$ to demonstrate the system level of readiness (see Figure 3). An annular linear induction pump (ALIP) was used to circulate NaK-78 through the loop of the TDU to transfer thermal energy from the reactor core simulator to a $12 \mathrm{kWe}$ free-piston Stirling power convertor. The $12 \mathrm{kWe}$ Stirling convertor is comprised of two dual-opposed 6 $\mathrm{kWe}$ Stirling engines. The core simulator heats the $\mathrm{NaK}$ to $550^{\circ} \mathrm{C}$, and as the heated $\mathrm{NaK}$ circulates through the loop, the thermal energy is transferred to the Stirling convertor through a pair of heat exchangers. The ALIP must impart a sufficient pressure rise on the $\mathrm{NaK}$ fluid, to overcome the pressure drop loss produced by the loop components (piping, core simulator, and the heat exchangers).
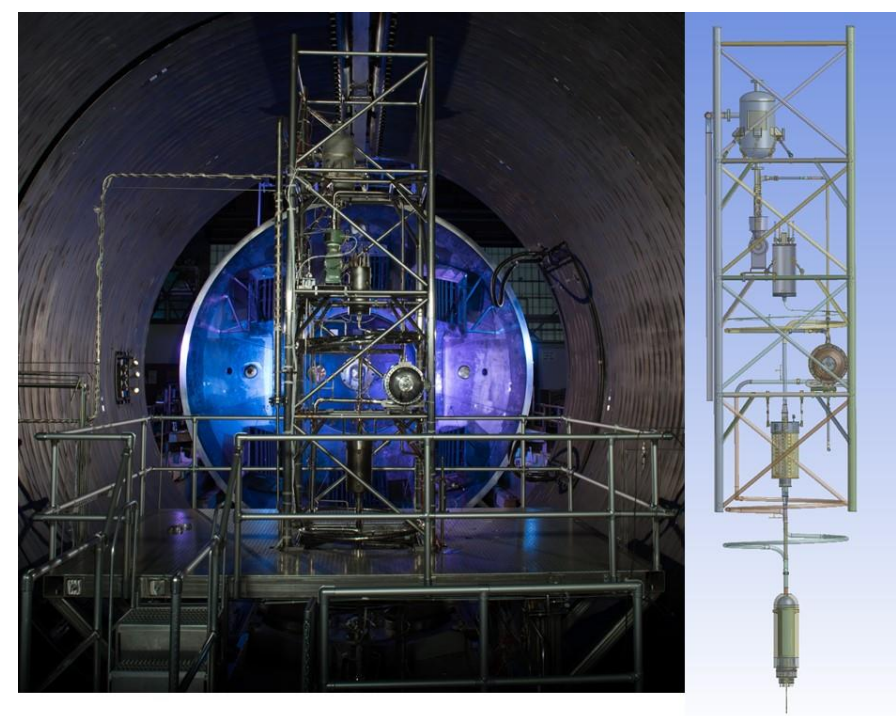

(a) Test article

(b) CAD Model

\section{Figure 3. Fission Power System (FPS) Technology Demonstration Unit (TDU).}

\section{Analysis Description}

A multi-physics two-step approach was taken to model the ALIP used in the TDU test. The following section describes the governing equations used by each solver in the various analyses. The application of these equations occurred in the magnetic and fluid flow solvers (ANSYS MAXWELL ${ }^{\circledR}$ and FLUENT ${ }^{\circledR}$ ). The conditions that were chosen for the calculations were based on data from experimental testing of the ALIP by the NASA Marshall Space Flight Center.

\section{A. Magnetic Field Analysis}

The ANSYS-Maxwell software was used to create a transient magnetic model of the ALIP. The Maxwell software basically solves the differential form of Maxwell's equations. The equations include 1) Faraday's Law of Induction, 2) Gauss's Law for magnetism, 3) Ampere's Law, and 4) Gauss's Law for electricity. The equations are as follows:

$$
\begin{aligned}
& \nabla \times E=-\frac{\partial B}{\partial t} \\
& \nabla \cdot \mathrm{B}=0 \\
& \nabla \times H=J+\frac{\partial D}{\partial t} \\
& \nabla \cdot D=\rho_{\text {charge }}
\end{aligned}
$$


The Maxwell transient field simulator calculates the time-domain magnetic fields in 3D. The source of the magnetic fields is the 3-phase current flowing through the 12 ALIP coils. The transient field simulator solves for the magnetic field $(\mathrm{H})$, current distribution $(\mathrm{J})$, and the magnetic flux density $(\mathrm{B})$. Derived quantities such as Lorentz force acting on the $\mathrm{NaK}$ fluid, winding flux linkage, and induced voltage/current in the NaK fluid are calculated from the basic field equations.

\section{B. Fluid Flow Field Analysis}

The flow field analysis involves the solution of the Navier Stokes equations, which include conservation of mass, momentum and energy. The conservation of mass (5) and conservation of momentum (6) equations are:

$$
\begin{aligned}
& \frac{\partial \rho}{\partial t}+\nabla *(\rho \vec{V})=C_{1} S_{m} \\
& \frac{\partial(\rho \vec{v})}{\partial t}+\nabla *(\rho \vec{V} \vec{V})=-\nabla P+C_{2} \nabla *(\overline{\bar{\tau}})+\rho \vec{g}+C_{3} \vec{F}
\end{aligned}
$$

The stress tensor, $\overline{\bar{\tau}}$, is given by

$$
\overline{\bar{\tau}}=\mu\left[\left(\nabla \vec{V}+\nabla \vec{V}^{T}\right)-\frac{2}{3} \nabla * \vec{V} I\right]
$$

where $\mu$ is the molecular viscosity, $I$ is the unit tensor, and the second term on the right hand side is the effect of volume dilation. The energy equation (8) is defined by

$$
\frac{\partial(\rho G)}{\partial t}+\nabla *(\vec{V}(\rho G+p))=\nabla\left(k \nabla T+* \boldsymbol{C}_{2}(\overline{\bar{\tau}} * \vec{V})\right)+\boldsymbol{C}_{4} S_{h}
$$

In the energy equation, $k$ is the thermal conductivity, while the three terms on the right hand side of the equation represent energy transfer due to conduction, viscous dissipation, and source/sink terms. It should be noted that there are source terms on the right-hand side of each of the above equations (Equations 5, 6, \& 8). Their coefficients are $C_{l}$ for mass, $C_{2}$ and $C_{3}$ for momentum, and $C_{4}$ for energy sources. These values are either 0 or 1 , depending on which source terms are used during the analysis. Two different approaches to solving the fluid flow problem were evaluated. The first approach was assumed to be incompressible and inviscid, $C_{1}=C_{2}=C_{3}=C_{4}=0$ (no viscous or source terms were used). In the second approach, the Boussinesq hypothesis (density changes due to buoyancy, which are accounted for in the realizable k- $\varepsilon$ turbulence model) was used so that $C_{l}=C_{4}=0$ and $C_{2}=C_{3}=1$ (viscous and source terms used). These constants are the basis for how the Lorentz forces from the magnetic analysis were handled relative to the CFD problem. This will be discussed in greater detail in the following section.

\section{Model Description}

ANSYS Design Modeler ${ }^{\circledR}$, Mesher, Maxwell ${ }^{\circledR}$, and FLUENT ${ }^{\circledR}$ were used to perform these analyses. The analyses were performed in series.

\section{A. Magnetic Field Model}

The 3D transient model is shown in Figure 4. The components modeled include the Hiperco 50 torpedo, two Hiperco 50 lamination half stacks, twelve copper coils, and the NaK-78 fluid contained within the body of the ALIP pump discretized as 25 individual segments or slugs. The 3D transient model takes advantage of symmetry. Only a 60 degree wedge of the ALIP was modeled to reduce computation time. Although the model was useful for calculating the magnetic forces acting on the liquid-metal contained within the ALIP, the predictions could not be compared directly with the experimental data.

Three-phase electrical power input is required to establish a moving magnetic wave down the annulus of the ALIP. Current amplitude and frequency are inputs for the

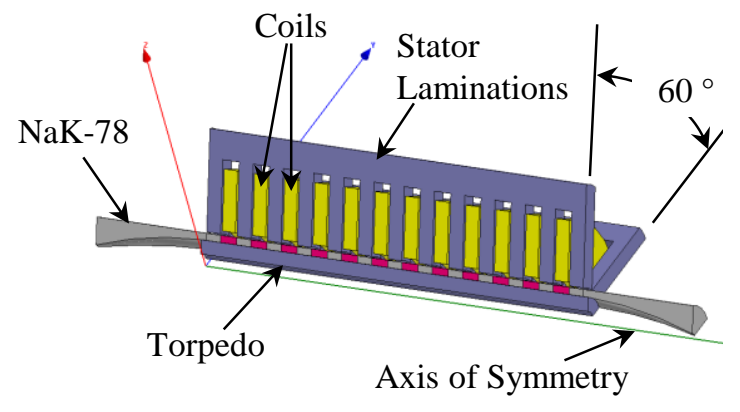

Figure 4. 3-D Transient Model of ALIP

American Institute of Aeronautics and Astronautics 
model. Figure 5 shows an example of the model input to simulate a $36 \mathrm{~Hz}, 18$ Ampere (peak) operating condition. To facilitate numerical stability in the transient solver, the current is ramped-up from zero to full current within two electrical cycles. Note that the current amplitudes of the three-phase power were not equal. During testing as MSFC, it was determined that for any given operating condition, the current in phase $B\left(I_{B}\right)$ was always greatest, while the phase A current $\left(\mathrm{I}_{\mathrm{A}}\right)$ was always the smallest with the phase $\mathrm{C}$ current $\left(\mathrm{I}_{\mathrm{C}}\right)$ falling in between. The current imbalance between the three phases was maintained for all model predictions for comparison with the MSFC data.

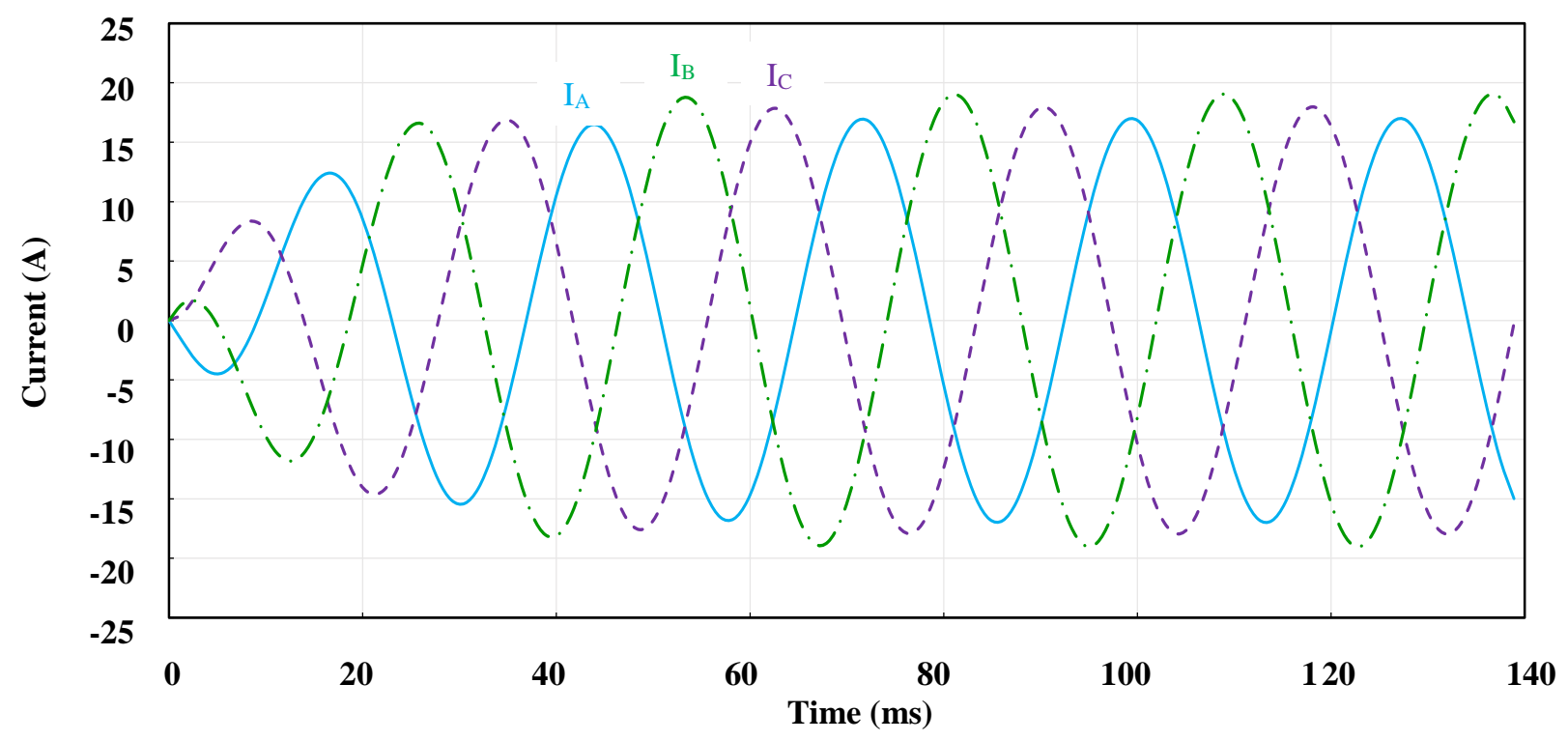

Figure 5. 3-Phase Current Ramp-up at $36 \mathrm{~Hz}$.

The transient magnetic model of the ALIP calculates the Lorentz forces and B-fields acting on each of the 25 slugs of NaK-78 fluid, for 5 complete electrical cycles (10 magnetic cycles). The 25 slugs of NaK fluid are shown in Figure 6. Slugs of $\mathrm{NaK}$ located adjacent to the stator poles are labeled beginning with the letter "P". Figures 7 and 8 show examples of the axial and radial Lorentz forces acting on a $\mathrm{NaK}$ slug adjacent to one of the stator poles (P2) over one electrical cycle, respectively. Note that the negative axial forces shown in Figure 7 oppose the NaK fluid flow in the pump. The radial forces shown in Figure 8 are always directed radially inward.

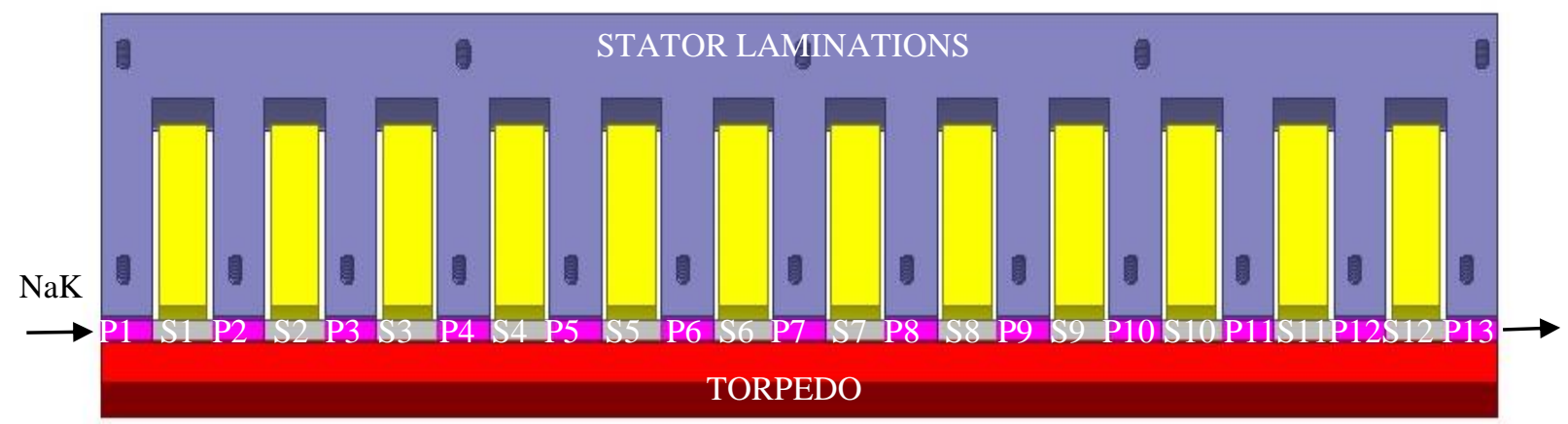

Figure 6. Section View of ALIP Defining NaK Slugs. 


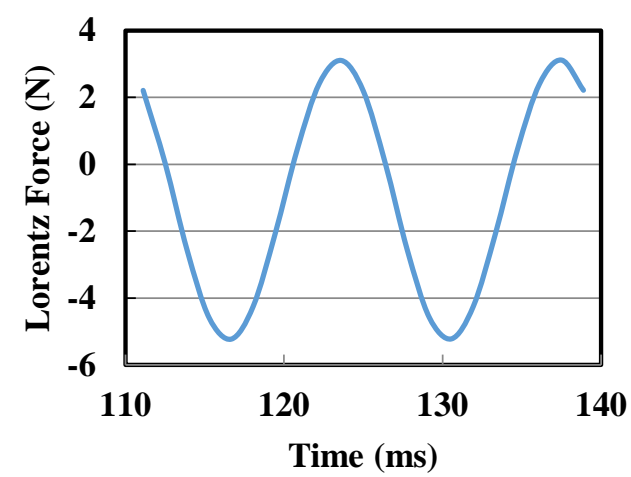

Figure 7. Axial Lorentz Forces acting on NaK Slug P2.

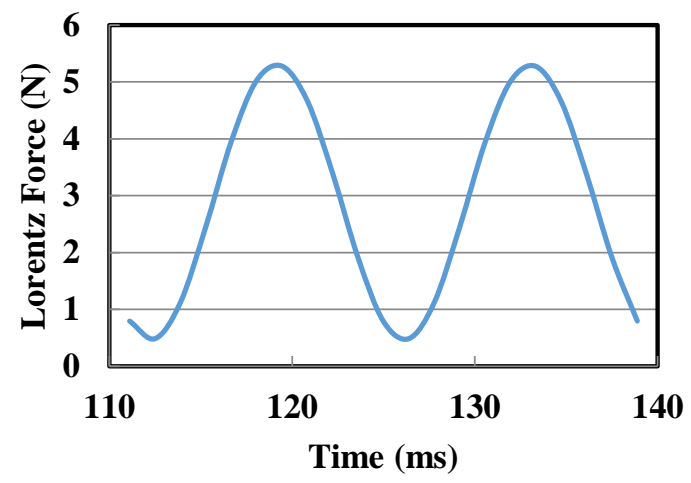

Figure 8. Radial Lorentz Forces acting on NaK Slug P2.

Figures 9 and 10 show the peak Lorentz Forces in the axial and radial directions for each NaK slug in the ALIP annulus over one electrical cycle. It is interesting to note that the majority of the axial force acting on the NaK is in alignment with the fluid flow direction. However, there are times when the axial force in portions of the pump oppose the fluid flow. Figure 10 shows that the radial forces acting on each NaK slug are always directed radially inward, and are generally larger than the axial forces. Based on these results, it can be concluded that the total (axial + radial) Lorentz forces acting on the $\mathrm{NaK}$ fluid are, in general, aimed more in the radially inward direction than in the axial direction.

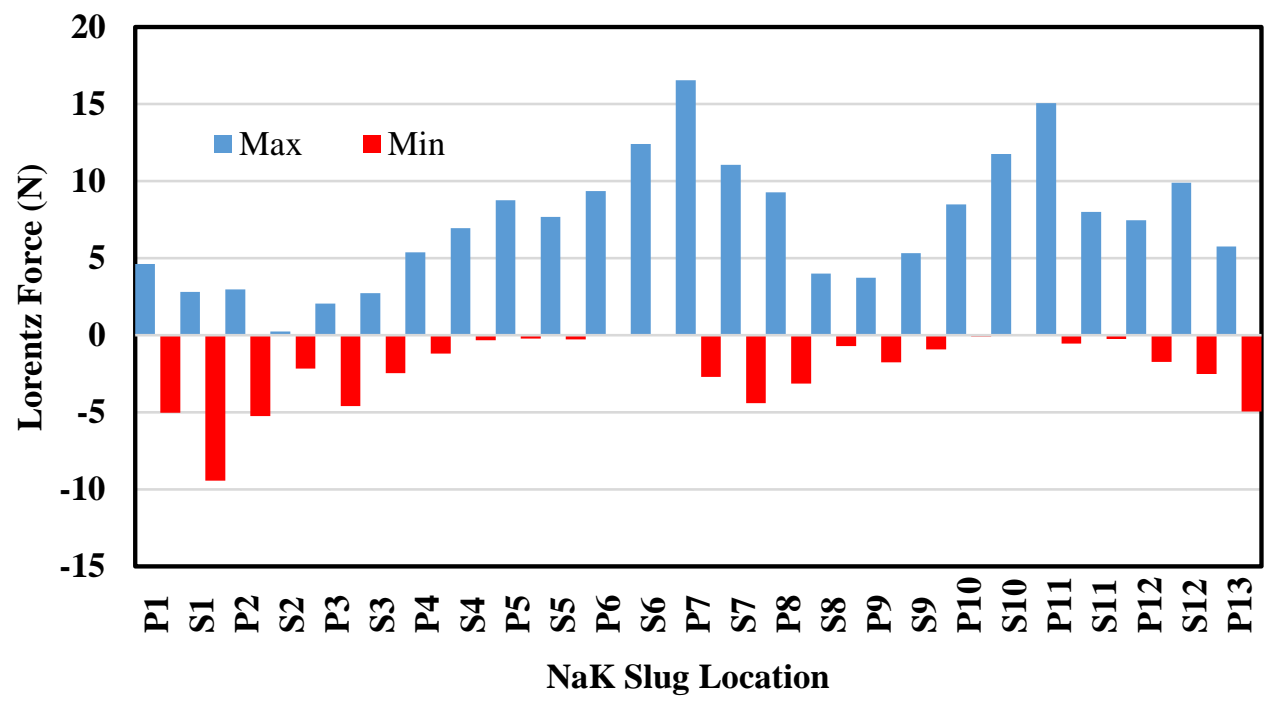

Figure 9. Peak Lorentz Forces acting on NaK Slugs in Axial Direction. 


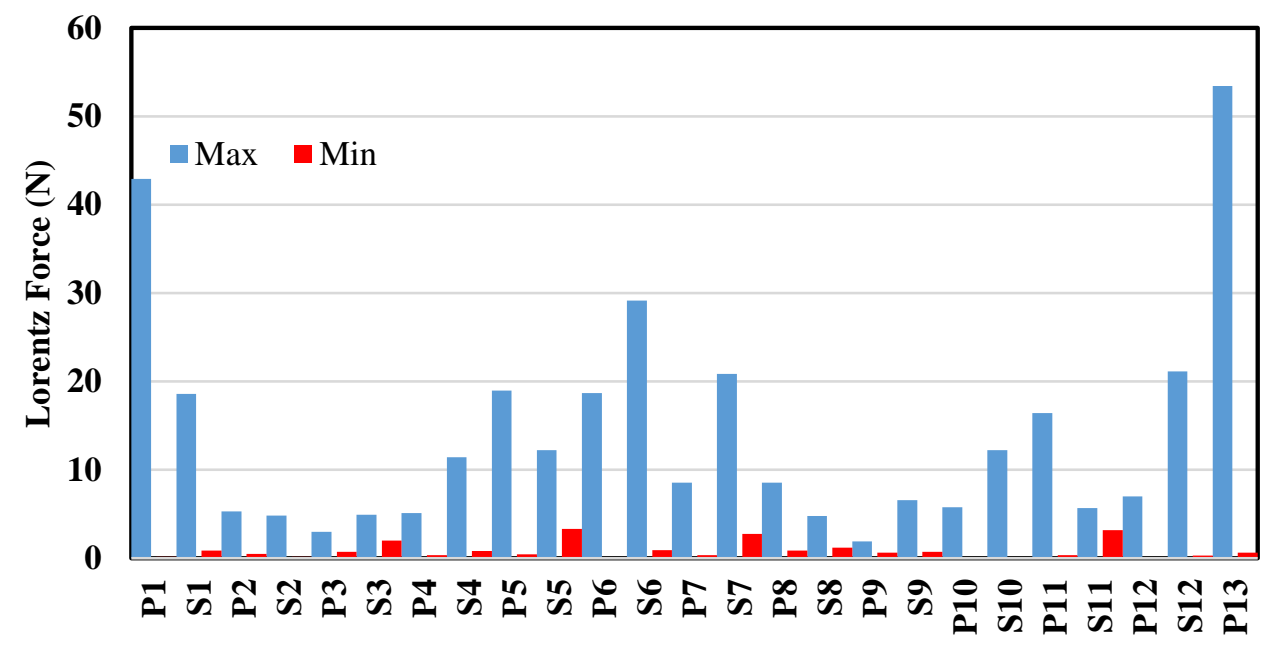

ALIP NaK Slug Location

Figure 10. Peak Lorentz Forces acting on NaK Slugs in Radial Direction

The prototypic ALIP pump used in the TDC test utilizes a hollow torpedo. The transient magnetic analysis was used to evaluate the torpedo to determine if there was sufficient material to carry the magnetic fields. Figure 11a shows the B-field magnitude in the torpedo body at an instant in time. Assuming a torpedo temperature of $550^{\circ} \mathrm{C}$, Figure 11a shows three locations (circled areas) were mild saturation may be occurring. According to GRC Hiperco $50 \mathrm{BH}$ measurements (shown in Figure 12), Hiperco 50 tends to saturate at about $1.8 \mathrm{~T}$ at $550^{\circ} \mathrm{C}$. The magnetic analysis was then repeated assuming a solid torpedo. Figure $11 \mathrm{~b}$ shows that the solid torpedo can eliminate the areas of saturation.

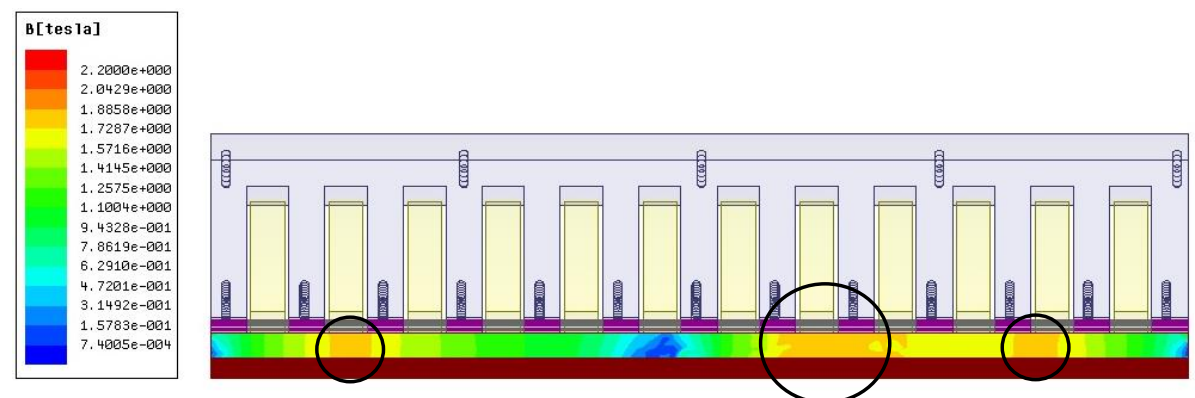

(a) B-field prediction for ALIP configured with hollow torpedo (baseline geometry)
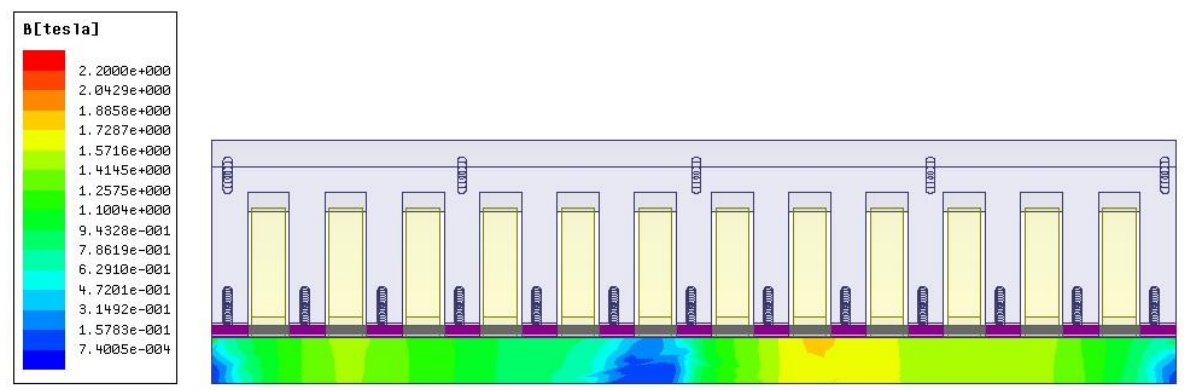

(b) B-field prediction for ALIP configured with solid torpedo

Figure 11. Torpedo B-field Magnitude at Time $=0.134722 \mathrm{~s}$

American Institute of Aeronautics and Astronautics 


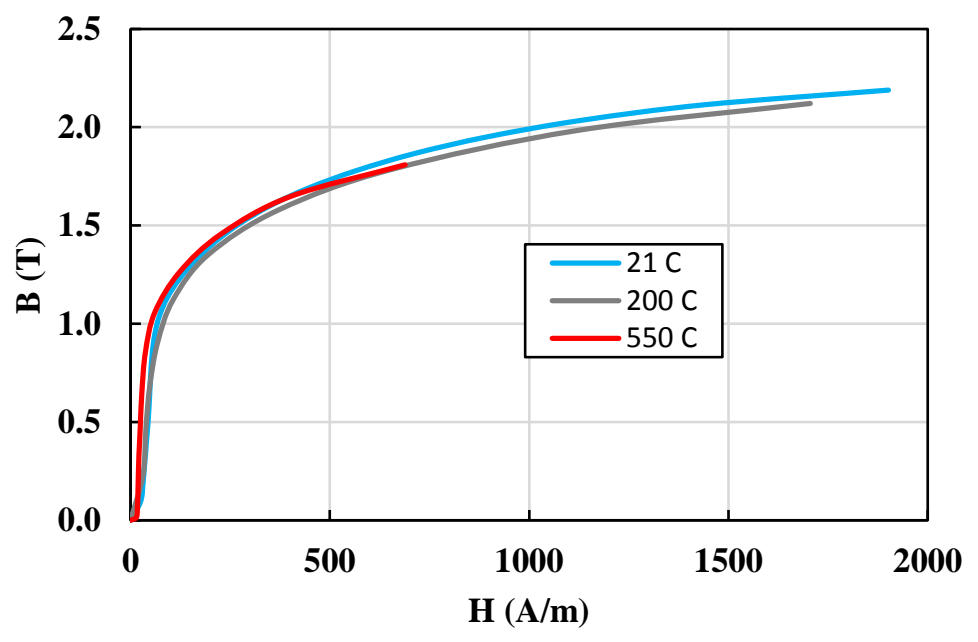

Figure 12. BH data for Hiperco 50 at various Temperatures.

\section{B. Computational Fluid Dynamics Model}

The geometry of the ALIP CFD model is shown in Figure 13. The CFD model includes the torpedo struts (supports torpedo in pump body as shown in Figure 13), and the slugs of $\mathrm{NaK}$ in the annulus. The solid torpedo volume was removed from the constant diameter mid-section, which forms the annulus. Inside the annulus are $13 \mathrm{NaK}$ slugs located adjacent to the stator poles, and $12 \mathrm{NaK}$ slugs located adjacent to the coils.

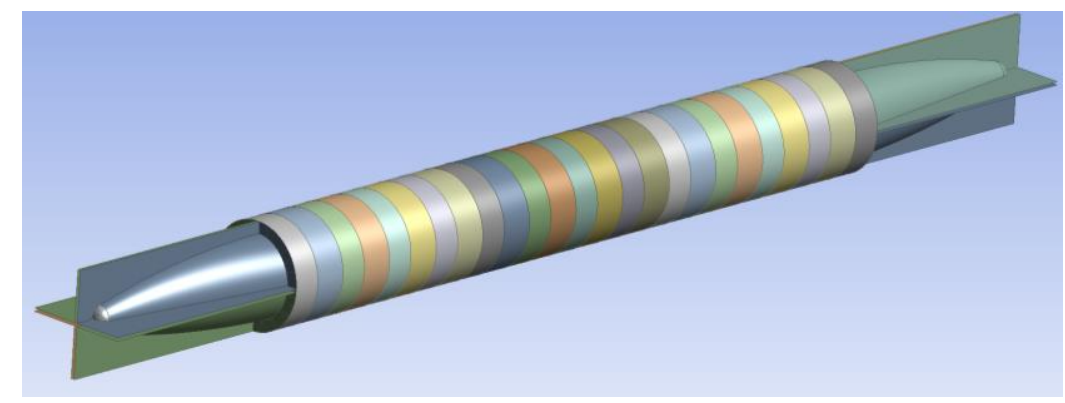

Figure 13. The ALIP CFD Model.

Figure 14 shows the target surfaces in the CFD model where Lorentz forces (produced by the 3D transient magnetic analysis model) are applied. As mentioned previously, two different CFD approaches were examined. In approach 1 , the results of the magnetic transient analysis are applied on two surfaces (axial and radial) for each of the 25 slugs of the CFD model. All of these transient force profiles are applied to the NaK in the annulus of the CFD model simultaneously, and calculations are performed until the CFD solution is time periodic (typically within a range of 5 to 10 cycles). In approach 2, the Lorentz forces are added to the source terms in the momentum equation.

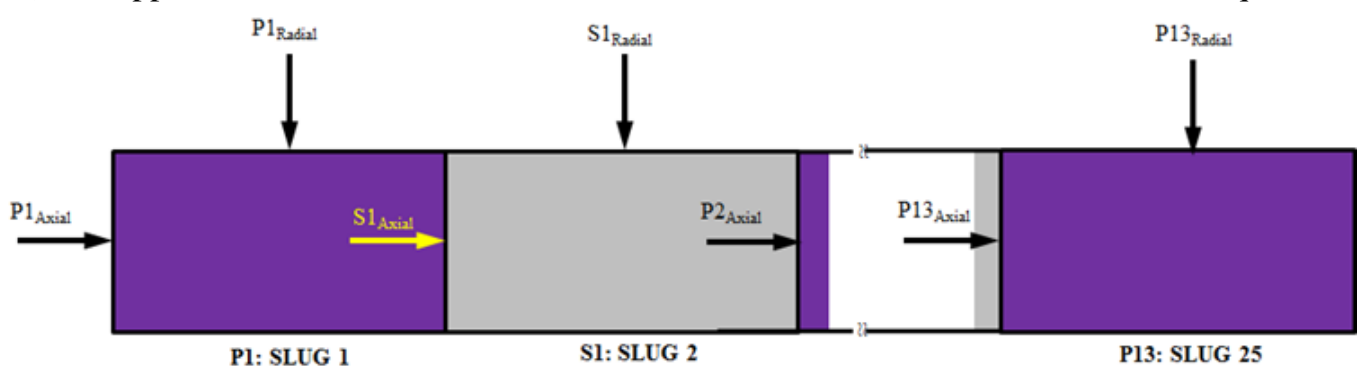

Figure 14. Orientation of NaK Slugs.

American Institute of Aeronautics and Astronautics 
The difference between the strategies is the method in which the Lorentz forces are applied to the NaK slugs. The equations that define the Lorentz forces are

where

$$
\vec{F}_{\mathrm{L}}=\vec{J} \times \vec{B}
$$

$$
\vec{J}=\sigma[\vec{E}+(\vec{V} \times \vec{B})]
$$

The variable $\mathrm{J}$ is the current distribution and $\mathrm{B}$ is the magnetic field. In approach 1 , the Lorentz forces $\left(\mathrm{F}_{\mathrm{L}}\right)$ are converted to pressures by dividing force by the area of application $\left(P \sim F_{L} / A_{\text {surface }}\right)$. The pressures acting on the $\mathrm{NaK}$ slug surfaces are updated every time step. Approach 2 involves adding the Lorentz forces as body force source terms $\left(\vec{F} \sim F_{L} / V l_{\text {slug }}\right)$ in the momentum equations ${ }^{5}$. Both strategies were evaluated in terms of ALIP pressure rise, and it was determined that approach 2 provided results that better matched the measured data. Therefore, the results and conclusions presented in this paper are based on the approach 2 strategy.

During the beginning stages of the calculations, consideration was given to including the throttling valve, which is a part of the ALIP test circuit (originally shown in Figure 2). A close-up view of the valve is shown in Figure 15. Its actual location in the ALIP Test Circuit is 15 to 20 diameters downstream of the ALIP exit and beyond a 90 degree bend. This valve provides a downstream resistance that affects both the operating pressure and the flow rate. Detailed measurements were made to determine the relationship between valve position and $\mathrm{NaK}$ flow rate. A plot of $\mathrm{NaK}$ flow rate with respect to valve position is shown in Figure 16.

Calculations were performed assuming approach 1 for the ALIP test circuit configured both with and without the valve. When in place, it is required that the valve be $91.5 \%$ closed to produce a volumetric flow rate of $\sim 60 \mathrm{GPM}$. The valve placement and resulting predicted NaK fluid velocities are shown in Figure 17. For these predictions, the valve was placed close to the exit of the computational domain to minimize the size of the mesh. However, when generating predictions with the valve in place, the separated flow downstream of the valve created an unsettled flow field that propagated out of the exit boundary in a non-uniform manner, which was deemed undesirable. The calculations were repeated with the valve removed, and the results were compared in Figure 18. It was discovered that the inclusion or omission of the valve in the model had no impact on the predicted pressure rise; the valve only affected the predicted ALIP inlet and outlet pressures. Unfortunately, including the valve in the model required far more computation time to reach a converged solution. As a result, it was decided to omit the valve from the simulations.

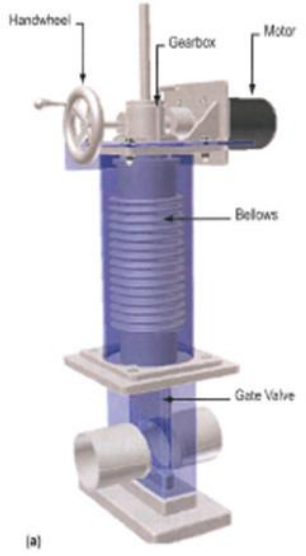

(a) Rendering

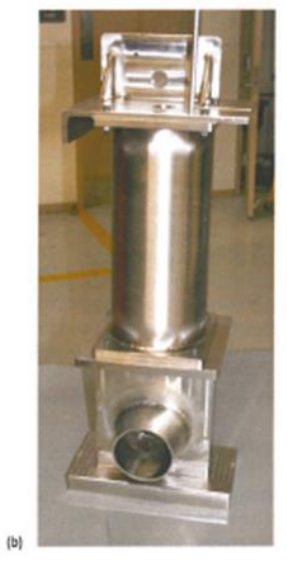

(b) Photograph

Figure 15. Throttling Valve used in the ALIP Test Circuit. (Courtesy of NASA Marshall Space Flight Center) 


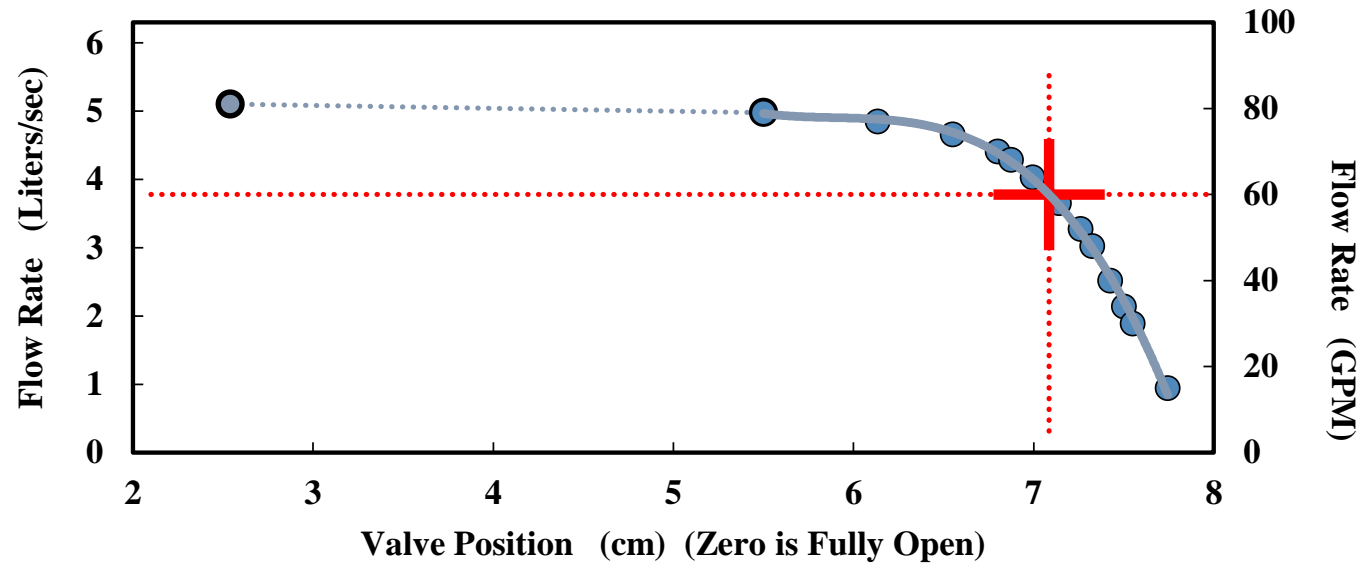

Figure 16. Characteristic Flow Curve of Custom Throttling Valve used in the ALIP Test Circuit. (Courtesy of NASA Marshall Space Flight Center)

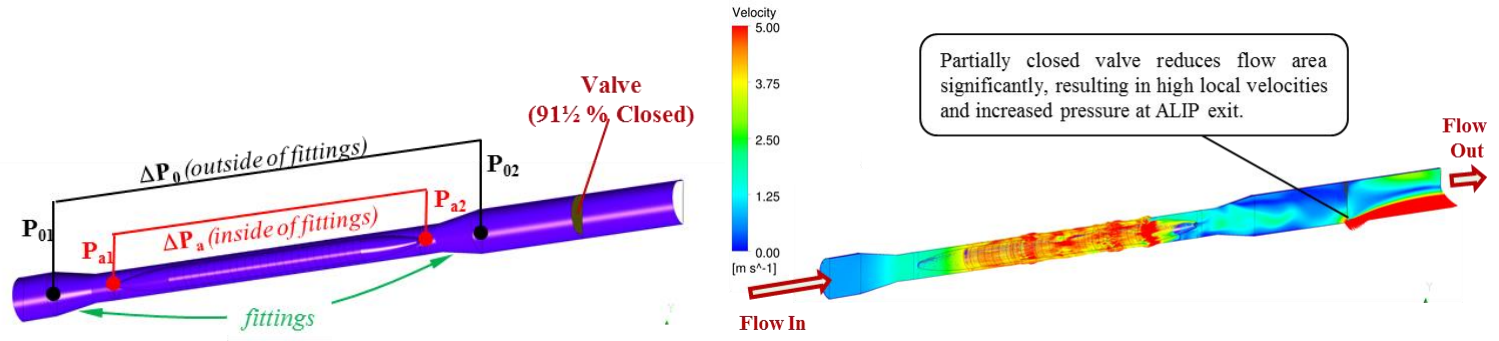
(a) Model
(b) Predicted Velocity Vectors

Figure 17. Partially Closed Gate Valve $(91.5 \%$ closed $)$ in the Computational Domain.
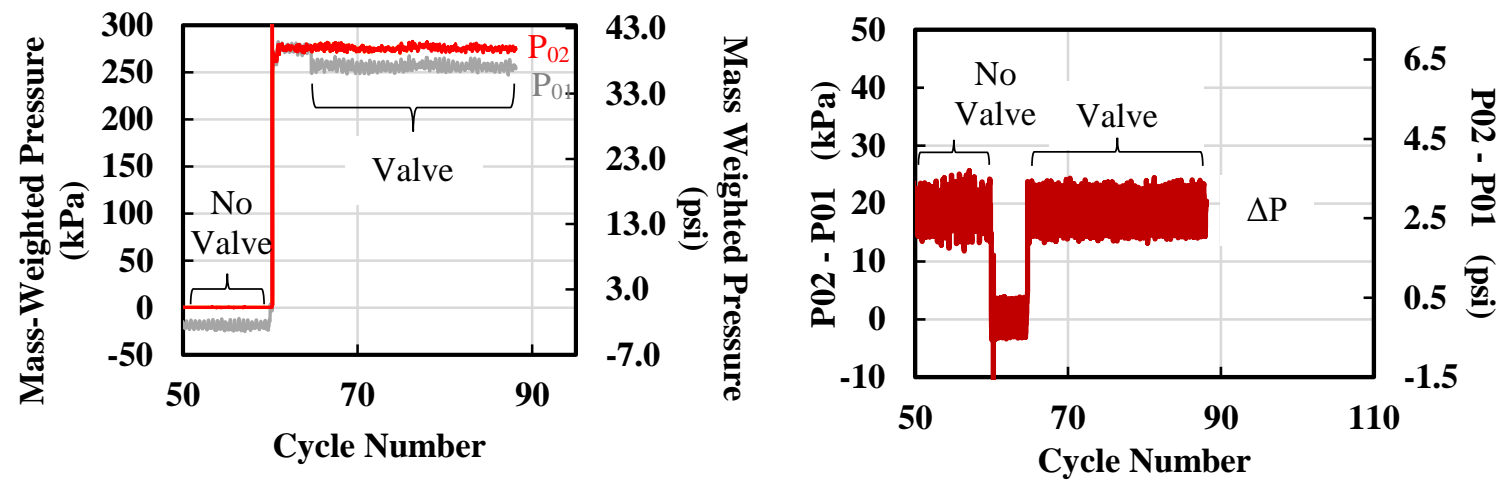

Figure 18. Baseline Predictions (60 GPM) with and without Downstream Valve. 


\section{Results}

As mentioned in section IV-B, two CFD approaches were evaluated. Approach $1\left(180^{\circ}\right.$ model) was used for only the baseline case. Once it was determined that the predicted pressure rise using this approach was about $15 \%$ lower than the measured value, it was decided to try approach 2 . However, approach $2\left(180^{\circ}\right.$ model) over-predicted the baseline measured data by $20 \%$. This computational over-prediction was also observed by Maidana ${ }^{5}$ when using reduced $\left(<360^{\circ}\right)$ CFD models that assumes symmetry. The approach 2 full $360^{\circ}$ model was considered the most accurate, and as a result, was used to generate the predictions shown in this paper.

Since the multi-physics simulation is computationally time intensive (it takes about a week to simulate a single test condition), a single flow rate (60 GPM) was chosen for code validation. At the selected validation point, the 3D multi-physics model predicted a pressure rise of $22.0 \mathrm{kPa}(3.2 \mathrm{psi}$ ) which was just slightly below (within $3 \%$ ) of the measured value of $22.6 \mathrm{kPa}$ (3.28 psi). The 3D multi-physics model was then used to predict the pump performance over a range of NaK flow rates as shown in Figure 19 and Table I. The predictions were in good agreement with the experimental data at the mid to high $\mathrm{NaK}$ flow rates. The agreement was not quite as good at the low NaK flow condition. The reason for this discrepancy may be due to the turbulence model being used in the CFD analysis. The $\mathrm{NaK}$ flow may not be fully turbulent at the low flow conditions. In addition, researchers have reported ${ }^{6}$ that all 2equation turbulence models (such as $\mathrm{k}-\varepsilon$ and $\mathrm{k}-\omega$ turbulence models) experience a reduction in accuracy in the presence of 'large' adverse pressure gradients. They tend to underpredict separation of the boundary layers due to this adverse pressure gradient. This leads to an underestimation of the effects of viscous-inviscid interaction which generally produces a performance estimate that is too optimistic for aerodynamic bodies. It is possible to live with this error if verification and validation indicates that it is not too high, otherwise a more robust turbulence model may be needed.

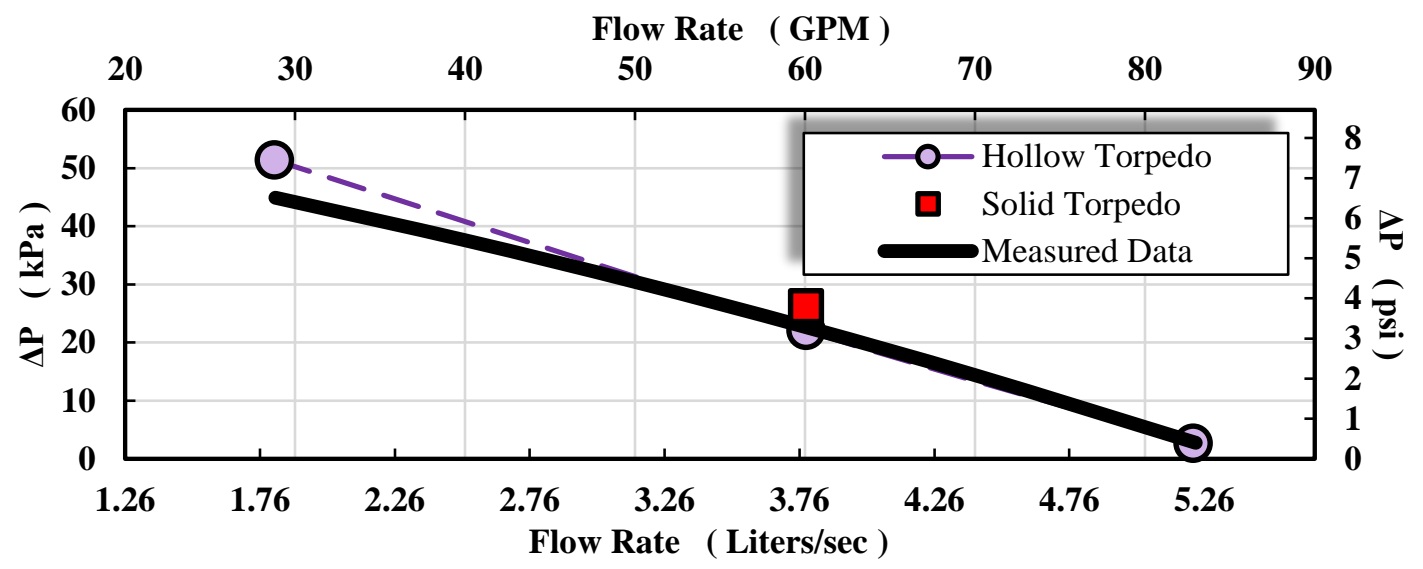

Figure 19. Comparison of Measured and Predicted ALIP Data (100 V, 18 A and $36 \mathrm{~Hz})$ (Measured Data Courtesy of NASA Marshall Space Flight Center).

Table I. Test Matrix Summary

\begin{tabular}{|c|c|c|c|c|c|c|c|}
\hline \multirow{2}{*}{$\begin{array}{l}\text { Torpedo } \\
\text { Configuration }\end{array}$} & \multicolumn{2}{|c|}{ Flow Rate } & \multicolumn{2}{|c|}{ Measured $\Delta P$} & \multicolumn{2}{|c|}{ Predicted $\Delta P$} & \multirow{2}{*}{$\begin{array}{c}\% \\
\text { Difference }\end{array}$} \\
\hline & GPM & Liter/sec & psi & $\mathbf{k P a}$ & psi & $\mathbf{k P a}$ & \\
\hline \multirow{3}{*}{ Hollow } & 28.8 & 1.82 & 6.51 & 44.87 & 7.45 & 51.37 & 14.5 \\
\hline & 60.0 & 3.79 & 3.28 & 22.61 & 3.20 & 22.03 & -2.6 \\
\hline & 82.9 & 5.23 & 0.40 & 2.76 & 0.38 & 2.64 & -4.4 \\
\hline Solid & 60.0 & 3.79 & & & 3.80 & 26.19 & $* 15.8$ \\
\hline
\end{tabular}

* \% difference relative to measurement recorded for 60 GPM in baseline ALIP (hollow torpedo) configuration

The multi-physics model was then used to explore ALIP geometric variations in an attempt to improve pump performance. At the time of this writing, only one design variation was completely modeled: a solid torpedo was substituted for the baseline hollow torpedo. When the hollow torpedo is replaced with a solid torpedo, the predicted pump pressure rise is $16 \%$ larger. This suggests that replacing the hollow torpedo with a solid torpedo would result 
in a modest performance improvement due to an increase in saturation margin. Another design variation that would be interesting to explore would be to substitute a stepped torpedo for the baseline hollow torpedo. The flow path of the NaK would still be a smooth annulus since the torpedo is encased inside a stainless steel sleeve. The diameter of the torpedo adjacent to the coils would be a little bit smaller than the diameter of the torpedo adjacent to the poles of the stator. The idea here is to encourage the B-field passing through the NaK to flow in a more radial direction, in hopes of better aligning the Lorentz Forces with the NaK flow direction. With the prototypic ALIP used in the TDU test, a simple substitution of a stepped torpedo for the hollow torpedo would probably not help since saturation would still be an issue. The whole ALIP pump would need to be redesigned to allow for a larger diameter torpedo to prevent B-field saturation.

\section{Conclusion}

The multi-physics approach described in this paper was found to be in good agreement with the ALIP experimental data at the mid to high flow operating conditions. Further model development is necessary to improve ALIP performance predictions over a wider range of operating conditions. As mentioned in section III-B, the realizable k$\varepsilon$ turbulence model was selected for use in the fluid flow analysis. It's possible that this turbulence model is less accurate at the pressure gradients encountered at the low NaK flow conditions. Several other turbulence models are available. Some effort should be applied toward determining the best turbulence model for use in this application.

Based on the results presented in this paper, the prototypic ALIP performance could potentially be improved by replacing the hollow torpedo with a solid configuration. In future ALIP designs, it might be beneficial to explore the impact of tailoring the torpedo geometry to better align the Lorentz forces with the NaK flow direction. The methods described in this paper may be of value in evaluating future ALIP designs.

\section{Acknowledgments}

This work was performed for the NASA Enabling Technology Development and Demonstration Program/Fission

Power Systems Project. The opinions expressed are those of the authors and do not necessarily reflect the views of NASA.

\section{References}

${ }^{1}$ Polzin, K. A., et al, "Performance Testing of a Prototypic Annular Linear Induction Pump for Fission Surface Power," NASA/TP-2010-216430, 2010.

${ }^{2}$ Geng, S. M., Niedra, J. M., Polzin, K. A., "Magnetic Analysis of an Annular Linear Induction Pump for Fission Power Systems," Proceedings of the Nuclear and Emerging Technologies for Space 2012 (NETS 2012), CP3033, ANSTD/ANS, Woodlands, TX, 2012.

${ }^{3}$ Fission Surface Power Team, "Fission Surface Power System Initial Concept Definition,” NASA/TM-2010-216772, 2010.

${ }^{4}$ Briggs, M. H., et al, "Fission Surface Power Technology Demonstration Unit Test Results," Proceedings of the Nuclear and Emerging Technologies for Space 2016 (NETS 2016), CP6074, ANS, Huntsville, AL, 2016.

${ }^{5}$ Maidana, C. O., Nieminen, J. E., "Multiphysics Analysis of Liquid Metal Annular Linear Induction Pumps: A Project Overview," Proceedings of the Nuclear and Emerging Technologies for Space 2016 (NETS 2016), CP6069, ANS, Huntsville, AL, 2016.

${ }^{6}$ Bardina, J.E., Huang, P.G., Coakley, T.J., "Turbulence Modeling Validation, Testing, and Development", NASA Technical Memorandum 110446, 1997. 